\title{
Comportamento Termoelástico de Vigas SMAHC sob Flexão em duas Temperaturas
}

\author{
Thermoelastic Behavior of SMAHC Beams \\ Subjected to Bending Loads at two Temperatures
}

Peter Faluhelyi, Flamínio Levy-Neto, Edson P. da Silva, Marcos V. Costa Sá,

Universidade de Brasília - UnB/FT/ENM CEP - 70910-900, Asa Norte, Brasília, DF e-mail: pyi@brturbo.com.br, flaminio@unb.br, dasilva@unb.br, marcuscsa@gmail.com

\section{RESUMO}

Neste trabalho, foi desenvolvido e caracterizado um compósito híbrido com memória de forma (SMAHC) por meio de uma análise teórico-experimental, no regime elástico, envolvendo a fabricação de vigas laminadas. A viga híbrida foi confeccionada em matriz de epóxi reforçada com fios de SMA e mantas de fibras picadas de vidro-E, com e sem bolsa de vácuo, de cura a frio e quente. A viga possui duas camadas de fios, com e sem roving, afastadas simetricamente o máximo possível do plano médio. Também foram produzidas vigas semelhantes, com e sem fios de aço galvanizado. Os módulos de elasticidade à flexão das vigas foram comparados aos módulos de simulações analíticas. $\mathrm{O}$ principal objetivo foi avaliar o aumento controlado da rigidez à flexão quando os fios de Ni-Ti (SMA) foram aquecidos, por efeito Joule, de $25^{\circ} \mathrm{C}$ a $69^{\circ} \mathrm{C}$. As vigas de SMAHC, com fração volumétrica de Ni-Ti até $2 \%$, tiveram um aumento no módulo de elasticidade de até $6,53 \%$, apenas mudando a fase dos fios de martensita $\left(25^{\circ} \mathrm{C}\right)$ para austenita $\left(69^{\circ} \mathrm{C}\right)$.

Palavras-chave: Liga Ni-Ti com memória de forma, viga SMAHC, comportamento termoelástico.

\begin{abstract}
In this paper, a shape memory alloy hybrid composite (SMAHC) was developed and characterized by theoretical and experimental analysis, in the elastic regime, involving the fabrication of laminated beams. The hybrid beam was made of epoxy matrix reinforced with SMA wires and E-glass chopped mat, with and without vacuum bag, as well as cold and hot curing. The two plies incorporating wires reinforced with roving or not, were symmetrically distributed along the thickness direction, as far as possible from the beam's middle surface. Similar specimens were also produced, substituting the Ni-Ti (SMA) wires by cheaper galvanized steel wires. The flexure elasticity modulus of the beams was compared with those obtained analytically. The main objective was to evaluate the bending stiffness of the beams, when the temperature of the Ni-Ti wires was increased by Joule effect, from $25^{\circ} \mathrm{C}$ and $69^{\circ} \mathrm{C}$. The SMAHC beams, with volume fraction of $\mathrm{Ni}-\mathrm{Ti}$ up to $2 \%$, presented an increase in the modulus, up to $6.53 \%$, by changing the phase of the wires, from the martensite $\left(25^{\circ} \mathrm{C}\right)$ to the austenite $\left(69^{\circ} \mathrm{C}\right)$.
\end{abstract}

Keywords: Ni-Ti shape memory alloy, SMAHC beam, thermoelastic behavior.

\section{INTRODUÇÃO}

A crescente demanda da sociedade e uma conjuntura favorável para o desenvolvimento de novos materiais viabilizaram um importante avanço tecnológico na área de estruturas adaptativas [1]. Atualmente, em alguns sistemas estruturais existe uma forte tendência à substituição de atuadores convencionais como motores elétricos; atuadores hidráulicos e pneumáticos que aumentam a complexidade e inviabilizam grandes reduções no peso global, por componentes adaptativos [1]. Neste contexto, JANOCHA [2] destacou o importante papel dos materiais inteligentes na inovação tecnológica, atuando, simultaneamente, como sensores e/ou atuadores, bem como componentes estruturais. Dentre esses materiais, encontram-se os piezoelétricos, magnetorestritivos e ligas com memória de forma (Shape Memory Alloy-SMA). Um novo cenário se apresenta para se criar estruturas autocontroladas, capazes de se adaptar a estímulos externos 
obtendo uma ou mais funções desejadas [2]. A vantagem tecnológica dos materiais funcionais em relação aos convencionais, é o comportamento peculiar da microestrutura atômica [3] . As SMA apresentam quatro temperaturas de transformação reversa de fase sólida (da Martensita para a Austenita) denominadas como: Temperatura de Formação Final da Martensita (Mf); Temperatura de Formação Inicial da Martensita (Ms), Temperatura de Formação Inicial da Austenita (As); e Temperatura de Formação Final da Austenita (Af). As ligas de Ni-Ti têm uma faixa de temperatura de transformação de $\mathrm{As}$ de $+166^{\circ} \mathrm{C}$ para $-50^{\circ} \mathrm{C}$, a medida que se altera a composição percentual de Níquel, em massa, de 54,5 \% a 56,5\%, respectivamente [4]. Adicionalmente, o módulo de elasticidade do fio de $\mathrm{Ni}$-Ti, na fase martensítica, EM $\left(25^{\circ} \mathrm{C}\right)$, praticamente dobra na fase austenítica $\left(69^{\circ} \mathrm{C}\right)$, assumindo o valor EA, i.e., EA=2.EM [1-4] . Ao longo dos últimos 50 anos, além dos modelos teóricos, os processos de fabricação e tratamentos para recuperação da memória de forma das SMA foram se aperfeiçoando, em especial, das ligas de Ni-Ti [ㄴ-ㅁ].

Em 1988, ROGERS E ROBERTSHAW [7] criaram um material compósito híbrido com memória de forma (Shape Memory Alloy Hybrid Composite-SMAHC), explorando as propriedades termoelásticas das SMA para aplicação no controle de vibrações acústicas. As descrições de leis constitutivas das SMA devem ser conhecidas para aplicação em sistemas estruturais, antes de ser alcançado o amplo potencial das estruturas adaptativas, enfatizado por BRISON et al. []]. Atualmente, o modelo constitutivo da Brinson [9] se destaca de outros, por representar melhor o comportamento termomecânico, em trabalhos teóricos e experimentais sobre vigas de compósitos poliméricos com filamentos de SMA embebidos ou fixados à superfície externa. Recentemente, surgiram novas propostas de desenvolvimento de vigas híbridas reforçadas com SMA, dentre elas, o trabalho de Turner 2001 [10]. Ele apresentou um modelo mais simplificado que o da Brinson e uma boa concordância entre resultados teóricos e experimentais, com aparatos instrumentais de grande precisão. A ideia central era obter uma previsão, com o novo modelo proposto, da máxima recuperação de forma de vigas SMAHC. Para isso, fabricou-se uma viga flexível, de comprimento de 558,8 mm e espessura de 1,9 mm, com 13,8 \% de fração volumétrica de SMA (em forma de lâminas) de cura em autoclave a vácuo $(0,6895 \mathrm{MPa})$, com temperaturas até $176,7^{\circ} \mathrm{C}$. As vigas foram laminadas com quatro camadas intercaladas uniformemente de $\mathrm{Ni}-\mathrm{Ti}$, na forma de cinco tiras contínuas lado a lado $(12,7 \mathrm{~mm})$ centralizadas na metade da largura da viga $(25,4 \mathrm{~mm})$. As tiras de $\mathrm{Ni}-\mathrm{Ti}$, pré-deformadas de 2 a $4 \%$, foram introduzidas na camada de $0^{\circ}$ dentro do tecido balanceado vidro-E pré-impregnado $\left(45^{\circ} / 0^{\circ} /-45^{\circ} / 90^{\circ}\right) 2 \mathrm{~s}$, todas embebidas em epóxi. Outros autores fabricaram vigas reforçadas com tecido pré-impregnado, de fibras de carbono ou aramida (Kevlar) quasi-isotrópicas, unidirecionais ou ortotrópicas, com distribuição uniforme de fios de SMA pré-deformados até $5 \%$, propondo o controle da forma e a redução de vibrações mecânicas ou acústicas para aplicações na indústria aeroespacial [11-16]. Existem também na literatura, vigas de matriz epóxi reforçada apenas com fios de SMA unidirecionais, sem e com pré-deformação de até 5\%, dispostos uniformemente na seção transversal, mostrando o aumento da rigidez, controladamente, resistência mecânica e das frequências naturais até o $3^{\circ}$ modo de vibração, quando aquecidos, por corrente elétrica [17-19]. Os compósitos híbridos, aquecidos por efeito Joule exigem maior cuidado na fabricação para que os fios de SMA fiquem isolados eletricamente entre si.

Neste trabalho exploratório, utilizando-se o dispositivo mostrado na Figura 1, foi dada continuação ao desenvolvimento, com processos diferentes de fabricação manual e caracterização de vigas SMAHC, com e sem bolsa de vácuo, de cura a frio e quente [20]. A seção transversal, simétrica, não-uniforme e composta de matriz epóxi reforçada com 2 camadas de fios de SMA, o mais afastadas possível do plano médio, dispostas entre 17 camadas de mantas isotrópicas de fibras de vidro-E, é ilustrada na Figura 2. E as camadas de fios foram laminadas com e sem reforço de fibras de vidro-E (roving) entre os fios. O desafio deste método proposto foi mostrar o aumento controlado da rigidez de um componente estrutural adaptativo de SMAHC, com uma fração volumétrica de até $2 \%$ de fios de SMA (Ni-Ti) embebidos, sem pré-deformação. Neste sentido, será testado o princípio da sintonização ativa de propriedades (Active Properties Tuning-APT) de Jia e Rogers [21]. Já que o módulo de elasticidade das SMA varia fortemente com a temperatura, para um aquecimento controlado, pode-se aumentar a rigidez de um sistema estrutural por meio de uma fonte de potência elétrica ligado a um dispositivo de controle da temperatura [22].

Os principais objetivos deste trabalho são: (I) avaliar a qualidade do método de fabricação de vigas prismáticas de SMAHC e (II) comparar, analítica e experimentalmente, os módulos de elasticidade em flexão de vigas de SMAHC com diferentes frações volumétricas de fios de $\mathrm{Ni}-\mathrm{Ti}$, a $25^{\circ} \mathrm{C}(\mathrm{T}<\mathrm{Mf})$ e a $69^{\circ} \mathrm{C}(\mathrm{T}>\mathrm{Af})$.

\section{MATERIAIS E MÉTODOS}

\subsection{Processo de Fabricação Manual de Vigas com e sem Fios Metálicos}


O aparato de fabricação é composto de um molde tipo macho-fêmea que possibilita a produção simultânea de até três vigas SMAHC. O molde permite instalação de duas camadas de fios metálicos, dispostas simetricamente em relação ao plano médio. Foi desenvolvido utilizando-se um perfil em U de aço como base de apoio do molde fêmea. A base de aço tem $1450 \mathrm{~mm}$ de comprimento, onde é fixado o dispositivo de estiramento de 16 furos espaçados uniformemente numa de suas extremidades. $\mathrm{O}$ molde fêmea de alumínio (perfil U) tem comprimento de $1220 \mathrm{~mm}$, largura interna de $31,3 \mathrm{~mm}$ e altura de $40 \mathrm{~mm}$. Ele foi fixado com blocos magnéticos na posição central interna do perfil de aço. E o molde macho de aço, de perfil vazado, tem comprimento $300 \mathrm{~mm}$ e seção quadrada de $31,2 \mathrm{~mm}$. Ele é pressionado para o interior do sulco do molde fêmea após a laminação da viga. Foram aparafusados os blocos-guias de alumínio no sulco do molde fêmea com distância entre eles, múltipla de $300 \mathrm{~mm}$ até $1200 \mathrm{~mm}$. Os blocos-guias de fios têm 8 furos (diâmetro de $1,2 \mathrm{~mm}$ ) espaçados regularmente ao longo do comprimento e seção quadrada de $9 \mathrm{~mm}$. Os suportes de fixação de fios foram instalados nas extremidades do perfil de aço. Eles são de aço com 8 furos (diâmetro de $1,5 \mathrm{~mm}$ ) espaçados regularmente $\mathrm{O}$ perfil $\mathrm{U}$, o molde fêmea, 6 blocos-guias e 2 suportes de fixação de fios podem ser visualizados na Figura 1.

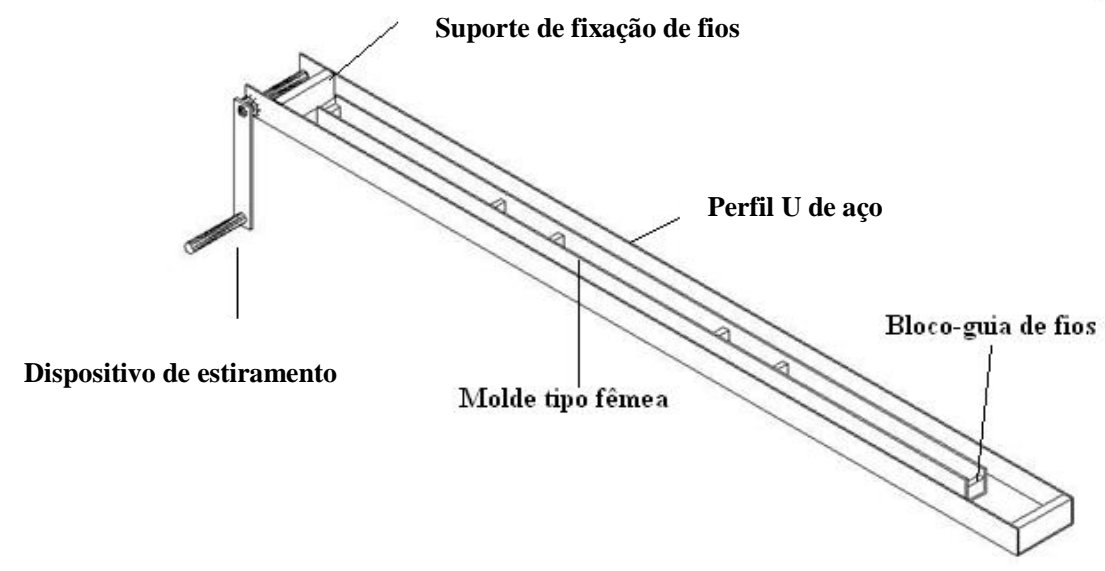

Figura 1: Bancada de fabricação das vigas compósitas com mantas de vidro-E e fios metálicos.

O procedimento de fabricação manual de vigas incluiu dois grupos com e sem bolsa de vácuo: (I) de cura a frio $\left(25^{\circ} \mathrm{C}\right)$ e (II) de cura a quente $\left(80^{\circ} \mathrm{C}\right.$ e $\left.121^{\circ} \mathrm{C}\right)$. O grupo (I) utilizou dois sistemas de resina epóxi de cura de 24 horas: LY1316/HY1208 $\left(\mathrm{Tg}=60^{\circ} \mathrm{C}\right)$ na camada de vidro-E/epóxi e Araldite-F/HY956 $\left(\mathrm{Tg}=80^{\circ} \mathrm{C}\right)$ na de fios. O grupo (II) utilizou um sistema de resina LY1316/HY1316 $\left(\mathrm{Tg}>80^{\circ} \mathrm{C}\right)$ de dois estágios de cura: de $10 \mathrm{~h}$ a $25^{\circ} \mathrm{C}$ seguido de $7 \mathrm{~h}$ de cura a quente $\left(80^{\circ} \mathrm{C}\right)$. No caso do grupo (II), o $1^{\circ}$ estágio, com vácuo $(0,064 \mathrm{MPa})$, ocorreu cobrindo-se a bancada, com bolsa de polipropileno transparente, vedada com massa de calafetar. E, no $2^{\circ}$ estágio, utilizou-se um sistema de placa aquecida, com temperatura controlada $\left(80^{\circ} \mathrm{C}\right)$. Ainda no grupo (II), foi realizado um processo mais sofisticado de cura, pela empresa Hot Bonder, com uma resina epóxi especial com cura de dois estágios: de $2 \mathrm{~h}$ a $80^{\circ} \mathrm{C}$ e de $2 \mathrm{~h}$ a $121^{\circ} \mathrm{C}$. Neste caso, o processo de fabricação empregou uma manta térmica de silicone com resistência elétrica interna ligada ao sistema HCS 9000B, que controlou a temperatura e o tempo de cura. O conjunto perfil $\mathrm{U}$ de aço-molde foi colocado sobre a manta térmica e coberto com bolsa de poliamida (nylon) transparente, vedada com fita selante (bam-bam), para fazer a cura a quente em dois estágios $\left(80^{\circ} \mathrm{C}\right.$ e $\left.121^{\circ} \mathrm{C}\right)$, simultaneamente com vácuo $(0,070 \mathrm{MPa})$.

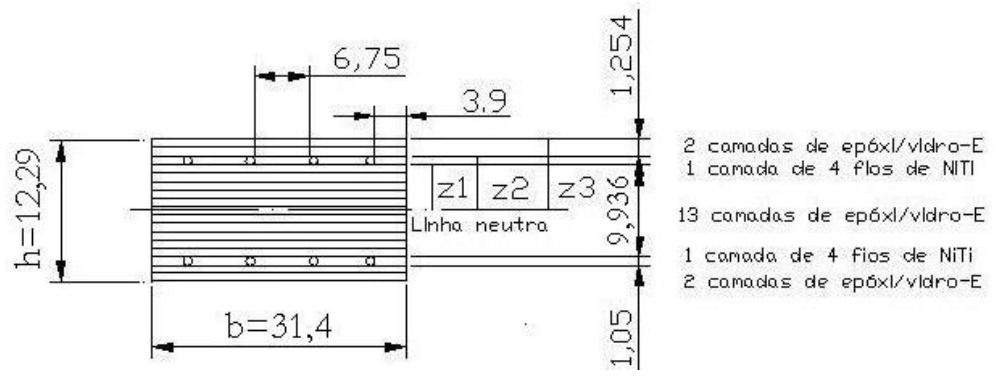

Figura 2: Seção transversal da viga de SMAHC (CP11) fabricada (dimensões em mm).

Na preparação das vigas compósitas, foi realizada a medição das massas dos materiais empregados, com uma 
balança da Balmak de resolução 1 g. Foi medida a massa dos fios, Mfio, das mantas fibras de vidro-E (de gramatura $450 \mathrm{~g} / \mathrm{m} 2$ ), Mv , e de roving (somente nos CP's 9, 10, 12, 13, 15 e 16), Mrov . Depois, calculou-se a massa de resina epóxi, Me . A massa de resina é preparada em função da massa de fibras, na proporção mínima de 1:1 [23]. E, devido à presença da camada de fios e às perdas provocadas pela pressão aplicada no molde macho, neste trabalho esta proporção foi de 1:1,5 [24]. Logo, a massa da resina preparada em cada caso (adesivo + endurecedor) foi determinada como $\mathrm{Me}=1,5 .(\mathrm{Mv}+\mathrm{Mrov})$. Foi aplicado no molde e no perfil de aço um desmoldante, cera Polywax. Os fios de Ni-Ti foram decapados com reagente Kroll [24]. Durante as laminações, os fios foram estirados e travados nos blocos-guia do dispositivo, visto na Figura 1. Os fios foram limpos com acetona industrial. No final, se aplicou uma pressão sobre o molde macho de 0,02 MPa. Após a cura, as vigas foram retiradas do molde e pesadas. Estes valores encontram-se na Tabela 1.

A viga SMAHC fabricada tem comprimentos (C) e larguras (b) nominais, C=300 $\mathrm{mm} \mathrm{e} \mathrm{b=31,4} \mathrm{mm,} \mathrm{e} \mathrm{uma}$ seção transversal típica é mostrada na Figura 2. Ao todo, foram confeccionados 16 corpos de prova (cp's) com as dimensões nominais mencionadas e espessuras (h) variadas. As 17 camadas de vidro-E/epóxi e as 2 camadas de fios/epóxi, com e sem roving, foram impregnadas manualmente com pincel, numa sequência ordenada: $2+1+13+1+2$, onde o " 1 " representa as camadas de fios. Fabricaram-se 7 cp's de SMAHC com 2 camadas de até 4 fios. Dentre eles, em 5 cp's foram adicionados rovings de vidro-E entre os fios, com duas tiras sobrepostas. O roving tem comprimento de $300 \mathrm{~mm}$, largura de $3 \mathrm{~mm}$ e espessura de $0,3 \mathrm{~mm}$.

\subsection{Caracterização das Vigas e dos Materiais Empregados}

A Tabela 1 mostra as principais características dos $16 \mathrm{cp}$ 's fabricados, onde se apresenta as espessuras, massa total e as frações volumétricas globais do vidro-E (mantas + roving, caso haja), Vgv, dos fios, Vfio , e do epóxi, Ve . Todas as frações volumétricas dos materiais, Vmat, foram calculadas por Vmat=(Mmat $/ \rho m a t) / v$, onde Mmat e $\rho$ mat são a massa e a densidade do material e o volume da viga, v=bhC. Sendo Mmat/ $/$ mat o volume de cada material ou fase da viga. O comprimento das vigas foi relacionado ao parâmetro C.

Tabela 1. Identificação e principais características das vigas compósitas com fios metálicos

\begin{tabular}{|c|c|c|c|c|c|c|c|}
\hline $\mathrm{CP}$ & Características & $\begin{array}{c}\text { Espessura } \\
\text { média } \\
(\mathrm{mm})\end{array}$ & $\begin{array}{c}\text { Desvio- } \\
\text { Padrão } \\
(\mathrm{mm}) \\
\end{array}$ & $\begin{array}{c}\text { Massa } \\
\text { Total } \\
(\mathrm{g}) \\
\end{array}$ & $\begin{array}{l}\mathrm{V}_{\mathrm{gv}} \\
\%\end{array}$ & $\begin{array}{l}\mathbf{V}_{\text {fio }} \\
\%\end{array}$ & $\begin{array}{l}\mathrm{V}_{\mathrm{e}} \\
\%\end{array}$ \\
\hline 1 & Sem fio, cura a frio, sem vácuo & 5,90 & 0,17 & 32 & 29,5 & ---- & 70,5 \\
\hline 2 & Sem fio, cura a frio, sem vácuo & 5,90 & 0,17 & 42 & 29,5 & ---- & 70,5 \\
\hline 3 & Sem fio, cura a frio, sem vácuo & $5 ; 90$ & 0,17 & 140 & 29,5 & ---- & 70,5 \\
\hline 4 & Sem fio, cura a frio, sem vácuo & 10,54 & 0,21 & 160 & 36,1 & ---- & 63,9 \\
\hline 5 & Sem fio, cura a frio, sem vácuo & 13,82 & 0,27 & 200 & 28,0 & ---- & 72,0 \\
\hline 6 & Sem fio, cura a quente, com vácuo & 7,52 & 0,17 & 121 & 45,5 & ---- & 54,5 \\
\hline 7 & 8 fios de aço, cura a frio, sem vácuo & 11,64 & 0,24 & 169 & 26,2 & 1,7 & 72,1 \\
\hline 8 & 8 fios de aço, cura a frio, sem vácuo & 15,14 & 0,54 & 227 & 27,8 & 1,3 & 70,9 \\
\hline 9 & $\begin{array}{l}4 \text { fios de } \mathrm{Ni}-\mathrm{Ti} \text {, roving (2 tiras), cura } \\
\text { a quente, com vácuo (Hot Bonder) }\end{array}$ & 8,93 & 0,22 & 131 & 38,4 & 1,2 & 60,4 \\
\hline 10 & $\begin{array}{l}6 \text { fios de } \mathrm{Ni}-\mathrm{Ti}, \text { roving (2 tiras), } \\
\text { cura a quente, com vácuo }\end{array}$ & 11,79 & 0,22 & 173 & 29,9 & 1,4 & 68,7 \\
\hline 11 & 8 fios de $\mathrm{Ni}$-Ti, cura a frio, sem vácuo & 12,29 & 0,37 & 180 & 22,5 & 1,8 & 75,7 \\
\hline 12 & 8 fios de $\mathrm{Ni}$ - $\mathrm{Ti}$, cura a frio, sem vácuo & 14,37 & 0,31 & 208 & 22,7 & 1,5 & 75,8 \\
\hline 13 & $\begin{array}{l}8 \text { fios de Ni-Ti, roving (2 tiras), } \\
\text { cura a frio, com vácuo }\end{array}$ & 13,76 & 0,14 & 203 & 26,4 & 1,6 & 72,0 \\
\hline 14 & $\begin{array}{l}8 \text { fios de Ni-Ti, cura a quente, } \\
\text { sem vácuo }\end{array}$ & 12,55 & 0,22 & 182 & 25,4 & 1,7 & 72,9 \\
\hline 15 & $\begin{array}{l}8 \text { fios de } \mathrm{Ni}-\mathrm{Ti} \text {, roving ( } 2 \text { tiras), } \\
\text { cura a quente, com vácuo }\end{array}$ & 11,41 & 0,15 & 174 & 30,1 & 1,9 & 68,0 \\
\hline 16 & $\begin{array}{l}8 \text { fios de } \mathrm{Ni}-\mathrm{Ti} \text {, roving (2 tiras), cura } \\
\text { a quente, com vácuo (Hot Bonder) }\end{array}$ & 12,40 & 0,13 & 178 & 28,8 & 1,8 & 69,4 \\
\hline
\end{tabular}


Para as fibras e resinas epóxi utilizadas, foram assumidas as propriedades fornecidas pelos fabricantes e obtidas na literatura [23-25], apresentadas na Tabela 2. Os fios de Ni-Ti na martensita e aço galvanizado, de $150 \mathrm{~mm}$ de comprimento útil, foram tracionados até a ruptura no laboratório da UnB, ensaiando-se inicialmente, a $25^{\circ} \mathrm{C}, 4 \mathrm{cp}$ 's de cada uma das ligas metálicas na máquina MTS-810. E, depois 2 cp's de fios de $\mathrm{Ni}-\mathrm{Ti}$ na austenita foram aquecidos controladamente até $69^{\circ} \mathrm{C}$, por efeito Joule, e mantida a esta temperatura para tração. Estes últimos cp's foram revestidos com isolamento elétrico nas extremidades, onde foram presos nos mordentes (40 mm de comprimento) ao serem fixados na MTS. Para isso, o fio foi envolvido com uma manta de vidro-E/epóxi curado a frio e sem bolsa de vácuo [20, 24] Os valores médios dos módulos dos fios de Ni-Ti na martensita e austenita, no regime elástico, foram adicionados à Tabela 2 , com diferenças percentuais respectivas de $-9,6 \%(25 \mathrm{GPa})$ e $-7 \%(50 \mathrm{GPa})$, em relação à literatura $[\underline{7}, 9]$. O fio de aço (baixo teor de carbono) revestido de zinco, com diâmetro 0,89 mm, modelo BWG 20 da Morlan. O fio de Ni-Ti da Memory Metalle, de extrusão a frio de $40 \%$ e tratamento térmico para recuperação da memória de forma (ou ativação de propriedades apenas). Além disso, conforme o manual do fabricante, os fios têm composição percentual em massa: 55,49\% de Ni, 44,50\% de Ti e 0,01\% de impurezas. E vem com a indicação na ficha técnica de $\mathrm{As}=62^{\circ} \mathrm{C}$. As temperaturas de transformação, que foram obtidas em um calorímetro diferencial de varredura (Diferencial Scanning Calorimeter-DSC) são: $\mathrm{Mf}=35,6^{\circ} \mathrm{C}, \mathrm{Ms}=48.7^{\circ} \mathrm{C}$, As $=46,7^{\circ} \mathrm{C}$ e $\mathrm{Af}=59,0^{\circ} \mathrm{C}$.

Tabela 2. Propriedades físicas e mecânicas dos materiais utilizados [요 24, 25]

\begin{tabular}{|c|c|c|c|}
\hline Material & $\begin{array}{c}\text { Densidade } \\
\left(\mathrm{g} / \mathrm{cm}^{3}\right)\end{array}$ & $\begin{array}{c}\text { Módulo de } \\
\text { Elasticidade } \\
(\mathrm{GPa})\end{array}$ & $\begin{array}{c}\text { Tensão de } \\
\text { Ruptura } \\
(\mathrm{MPa})\end{array}$ \\
\hline Fio de aço galvanizado & 6,37 & 75,5 & 500 \\
\hline Fibras de vidro-E & 2,54 & 70 & 2400 \\
\hline Resina epóxi & $1,05-1,20$ & 3,5 & 55 \\
\hline Fio de Ni-Ti (martensita) & 6,45 & 22,6 & 750 \\
\hline Fio de Ni-Ti (austenita) & 6,45 & 48,4 & 930 \\
\hline
\end{tabular}

\subsection{Sistema de controle da temperatura dos fios de $\mathrm{Ni}-\mathrm{Ti}$}

O sistema de controle de temperatura da viga adaptativa foi montado com um termopar (tipo J), um amplificador AD594AQ (saída calibrada em $10 \mathrm{mV} /{ }^{\circ} \mathrm{C}$ ), um PIC $18 \mathrm{~F} 452$ (microcontrolador) e um relé, esquematizados na Figura 3. Este sistema básico, chamado de controle ON-OFF, mantém a corrente elétrica para a viga até a temperatura de operação de $69^{\circ} \mathrm{C}$ [24]. Nesta temperatura, o relé desliga automaticamente a fonte de potência elétrica, de $135 \mathrm{~W}$ (30 V D.C.), que fornece uma corrente elétrica (Imax=4,5 A) aos fios de $\mathrm{Ni}$-Ti. Os fios estão ligados, em série, por conectores nas extremidades da viga, que atua como resistor no circuito elétrico. O sistema de controle de liga-desliga da fonte foi ajustado para uma variação de temperatura de aquecimento das vigas entre $68^{\circ} \mathrm{C}$ e $71^{\circ} \mathrm{C}$. Por precaução, um termômetro digital, modelo ET 1400 (resolução de $1^{\circ} \mathrm{C}$ ) da Minipa, com um termopar tipo $\mathrm{J}$, monitorou a temperatura da superfície das vigas para garantir a segurança nos ensaios de flexão na MTS-810.

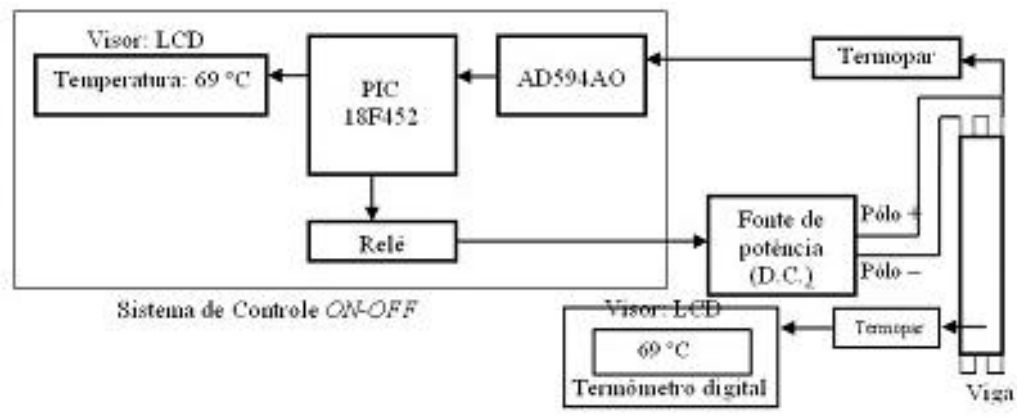

Figura 3: Esquema do sistema de controle $O N-O F F$ da fonte de potência ligado à viga adaptativa [23]

\subsection{Modelo de Euler-Bernoulli de Vigas SMAHC sob Flexão}

No modelo teórico, que relaciona a flecha com a força aplicada nos ensaios de flexão de três pontos, foi utilizada a Teoria Clássica de Laminados, para vigas de Euler-Bernoulli [23-25]. Neste caso, cabe ressaltar que a razão entre o vão, L, nos ensaios e a espessura das vigas, h, deve ser igual ou superior a 10, i.e. L/h $\geq 10$. 
A seção transversal, de 19 camadas, tem disposição das duas camadas de fios, simétricas em relação ao plano médio, mostrada na Figura 4. A Eq. (1) mostra o polinômio que melhor se aproxima da curva de Eev versus Vv de Mendonça [25], a qual foi obtida utilizando-se a Teoria Clássica de Laminados [23] e o fato de que as lâminas de manta de vidro-E/epóxi são isotrópicas. A Eq. (2) baseia-se na regra das misturas, da Teoria Micromecânica Clássica. A Eq. (3), na qual está implícito que no plano médio da viga z0=0, refere-se ao módulo de rigidez à flexão de uma viga isotrópica equivalente [23]. As equações (1) a (3) são consideradas boas aproximações na modelagem do comportamento elástico de lâminas e laminados compósitos [르, 25].

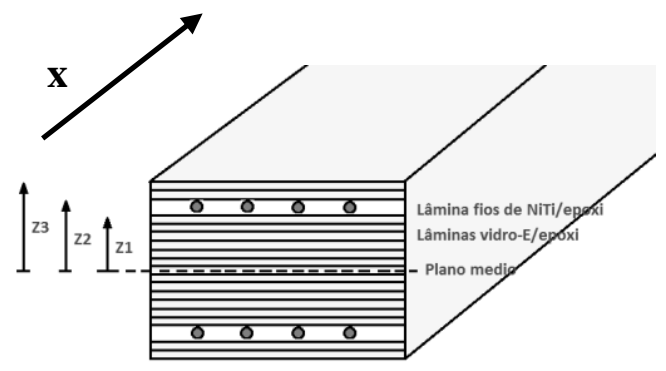

Figura 4: Configuração geral da concepção proposta da viga compósita híbrida com fios metálicos.

$$
\begin{aligned}
& E_{e v}=3,44+28,2 V_{v}+21,6 V_{v}^{4} \\
& E_{2}=E_{e} V_{e 2}+E_{r o v} V_{r o v}+E_{f i o} V_{f i o 2} \\
& E_{f}=\frac{8}{h^{3}}\left[E_{1} z_{1}^{3}+E_{2}\left(z_{2}^{3}-z_{1}^{3}\right)+E_{3}\left(z_{3}^{3}-z_{2}^{3}\right)\right]
\end{aligned}
$$

A fração volumétrica de mantas de vidro-E, Vv , foi empregada na Eq. (1), que fornece o módulo elástico das camadas (lâminas) sem fios. Para as camadas de fios usa-se a Eq. (2), onde as frações volumétricas de epóxi, roving e fios são dadas por, Ve2, Vrov e Vfio2, respectivamente, e o volume destas camadas é v2=bdC. Quando não se usou roving, Vrov=0, o segundo termo da Eq. (2) foi suprimido.

$\mathrm{Na}$ Equação (3), que fornece o módulo em flexão das vigas, as distâncias máximas de afastamento em relação ao plano médio das vigas: do núcleo de vidro-E/epóxi; da camada com fios; e das duas camadas externas, são dadas respectivamente por z1 , z2=z1+d e z3=h/2. Definiu-se $z 1=\left(\left(N^{\prime}-4\right) / 2\right)$.hev , onde hev=(h$2 \mathrm{~d}) / \mathrm{N}^{\prime}$ é a espessura estimada da lâmina de epóxi/vidro-E, e $\mathrm{N}^{\prime}=\mathrm{N}-2$ o número de camadas do laminado $(\mathrm{N})$ subtraído das duas camadas de fios. Para todos os cp's com fios, foi escolhido $\mathrm{N}=19$, notando que em z1 se considerou $\mathrm{N}^{\prime}=17$, obtido subtraindo-se as duas camadas mais externas em relação ao plano médio. Para os cp's sem fios, os números de camadas foram: $\mathrm{N}=10$ (CP's 1, 2 e 3); N=21 (CP6); e N=23 (CP's 4 e 5).

Os ensaios, em regime elástico, seguiram a norma ASTM D-790-07 para vigas compósitas, submetidas à flexão de três pontos (Fig. 5), nas temperaturas de $25^{\circ} \mathrm{C}$ e $69^{\circ} \mathrm{C}$. Os ensaios foram realizados na máquina universal MTS-810, com célula de carga com capacidade de $10 \mathrm{kN}$ e resolução de $100 \mathrm{~N}$. A MTS-810 foi programada para uma taxa de deslocamento de $1 \mathrm{~mm} /$ minuto, com deflexão máxima das vigas de $1 \%$ do vão entre apoios escolhido. Os vãos utilizados foram: $114 \mathrm{~mm}, 150 \mathrm{~mm}, 280 \mathrm{~mm}$ e $467 \mathrm{~mm}$.

Um cilindro de aço foi utilizado para aplicar a carga, monotonicamente, no ponto médio dos cp's e distribuída ao longo de toda a largura deles. Os ensaios de vigas SMAHC, com o sistema de aquecimento controlado conectado à viga, foram realizados na temperatura de $69^{\circ} \mathrm{C}$. O CP13 na fase austenita, sob flexão na MTS-810, é mostrado na Figura 5. 


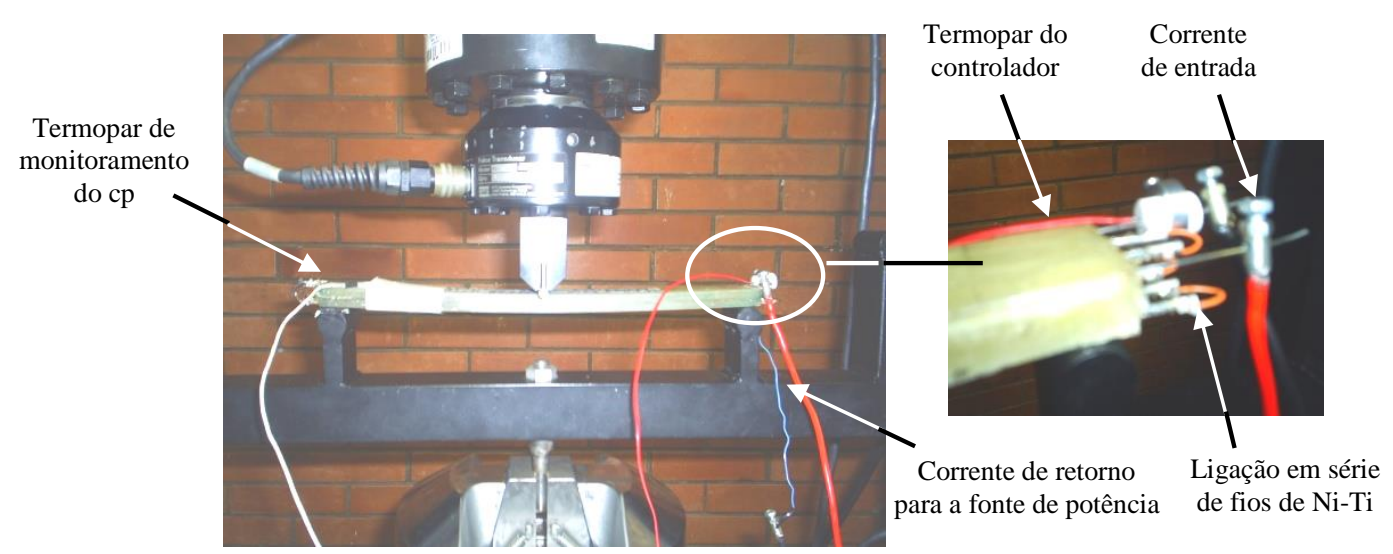

Figura 5: Viga SMAHC sob flexão para vão $\mathrm{L}=280 \mathrm{~mm}$ à temperatura de $69^{\circ} \mathrm{C}>\mathrm{A}_{\mathrm{f}}[\underline{25}$ ].

\section{RESULTADOS TEÓRICOS E EXPERIMENTAIS DE VIGAS SOB FLEXÃO}

A norma ASTM D-790-07 recomenda para determinação dos módulos de elasticidade à flexão de vigas compósitas biapoiadas uma razão de $\mathrm{L} / \mathrm{h} \geq 16$. Os módulos experimentais à flexão (E) para $25^{\circ} \mathrm{C}$ e $69^{\circ} \mathrm{C}$ foram determinados com base na Teoria Clássica de Euler-Bernoulli, utilizando-se a Equação (4) [23-25]:

$$
E=P L^{3} \cdot(48 I \delta)^{-1}
$$

Tabela 3: Módulos analíticos e experimentais à flexão para quatro diferentes vãos (L) em duas temperaturas

\begin{tabular}{|c|c|c|c|c|c|c|c|c|c|}
\hline \multirow{3}{*}{$\mathrm{CP}^{* * * *}$} & \multicolumn{5}{|c|}{ Módulo de Elasticidade (GPa) } & \multirow{2}{*}{\multicolumn{4}{|c|}{$\begin{array}{c}\text { Diferença Percentual }^{* *} \\
(\%)\end{array}$}} \\
\hline & \multirow{2}{*}{$\begin{array}{c}\text { Analítico } \\
\left(\mathrm{E}_{\mathrm{anal}}\right)\end{array}$} & \multicolumn{4}{|c|}{$\begin{array}{c}\text { Experimental }\left(\mathrm{E}_{\exp }\right) \\
\text { (para diferentes vãos, } \mathrm{L})\end{array}$} & & & & \\
\hline & & $114^{*}$ & $150^{*}$ & $280^{*}$ & $467^{*}$ & $114^{*}$ & $150^{*}$ & $280^{*}$ & $467^{*}$ \\
\hline 1 & 11,94 & 10,51 & ------ & ------ & ------ & $+13,61$ & $\begin{array}{c}----- \\
\end{array}$ & ------ & ------ \\
\hline 2 & 11,94 & ------ & 10,68 & 11,71 & ------ & ------ & $+11,80$ & $+1,96$ & ------ \\
\hline 3 & 11,94 & ------ & ------ & ------ & 11,90 & $\begin{array}{ll}----- \\
\end{array}$ & ------ & ------ & $+0,34$ \\
\hline 4 & 13,97 & ------ & 13,64 & 13,81 & ------ & ------ & $+2,42$ & $+0,34$ & ------ \\
\hline 5 & 11.46 & 9,58 & 10,39 & 11,41 & ------ & $+19,62$ & $+10,30$ & $+1,16$ & ------ \\
\hline 6 & 17,21 & ------ & 16,24 & 17,17 & ------ & ------ & $+5,97$ & $+0,23$ & ------ \\
\hline 7 & 10,53 & 8,55 & 10,09 & 10,52 & ------ & $+23,16$ & $+4,36$ & $+0,10$ & ------ \\
\hline 8 & 11,16 & ------ & ------ & 10,90 & ------ & ------ & ------ & $+2,39$ & ------ \\
\hline $9 \mathrm{M}$ & 16,15 & ------ & 13,79 & 16,01 & ------ & ------ & $+17,11$ & $+0,87$ & ------ \\
\hline $9 \mathrm{~A}$ & 16,62 & ------ & 14,02 & 15,67 & ------ & ------ & $+18,54$ & $+6,06$ & ------ \\
\hline $10 \mathrm{M}$ & 14,01 & ------ & 12,28 & 13,98 & ------ & ------ & $+14,09$ & $+0,21$ & ------ \\
\hline $10 \mathrm{~A}$ & 14,57 & ------ & 12,14 & 13,30 & ------ & ------ & $+20,02$ & $+9,55$ & ------ \\
\hline $11 \mathrm{M}$ & 9,53 & 8,40 & ------ & 9,50 & ------ & $+13,45$ & ------ & $+0,32$ & ------ \\
\hline $11 \mathrm{~A}$ & 10,25 & 9,37 & ------ & 10,12 & ------ & $+9,39$ & ------ & $+1,28$ & ------ \\
\hline $12 \mathrm{M}$ & 10,02 & ------ & 9,43 & 9,77 & ------ & ------ & $+6,26$ & $+2,56$ & ------ \\
\hline $12 \mathrm{~A}$ & 10,83 & ------ & 9,25 & 9,91 & ------ & ------ & $+17,08$ & $+9,28$ & ------- \\
\hline $13 \mathrm{M}$ & 13,44 & ------ & 11,64 & 13,01 & ------ & ------ & $+15,46$ & $+3,31$ & ------ \\
\hline $13 \mathrm{~A}$ & 14,09 & ------ & 12,10 & 12,63 & ------ & ------ & $+16,45$ & $+1,56$ & ------ \\
\hline $14 \mathrm{M}$ & 10,59 & 8,60 & ------ & 10,57 & ------ & $+23,14$ & ------ & $+0,19$ & ------ \\
\hline $14 \mathrm{~A}$ & 11,57 & 9,38 & ------ & 10,03 & ------ & $+23,35$ & ------ & $+15,35$ & ------ \\
\hline $15 \mathrm{M}$ & 14,25 & ------ & 12,84 & 13,98 & ------ & ------ & $+10,98$ & $+1,93$ & ------ \\
\hline $15 \mathrm{~A}$ & 15,02 & ------ & 12,88 & 12,95 & ------ & ------ & $+16,61$ & $+15,98$ & ------ \\
\hline $16 \mathrm{M}$ & 13,80 & ------ & 11,64 & 13,61 & ------ & ------ & $+18,56$ & $+1,40$ & ------ \\
\hline $16 \mathrm{~A}$ & 14,52 & ------ & 11,66 & 11,21 & ------ & ------ & $+24,53$ & $+29,53$ & ------ \\
\hline
\end{tabular}

"Os cp's foram ensaiados para os vãos entre apoios (L) apresentados com medidas em $\mathrm{mm}$.

${ }^{* *}$ As diferenças percentuais foram calculadas pela fórmula: $\left[\left(\mathrm{E}_{\text {anal }}-\mathrm{E}_{\mathrm{exp}}\right) / \mathrm{E}_{\exp }\right] \mathrm{x} 100$.

${ }^{* * *} \mathrm{Os}$ cp's com fios de Ni-Ti na martensita e austenita são identificados com as letras M e A, respectivamente.

O vão entre apoios é L, o momento de inércia da área da seção transversal, I=(bh3)/12, a carga aplicada no 
centro $(\mathrm{x}=\mathrm{L} / 2)$ da viga, $\mathrm{P}$, e a deflexão, $\delta$, no mesmo ponto. Na Tabela 3, são apresentados os módulos experimentais das vigas compósitas, submetidas à flexão, com flecha máxima de $1 \%$ do vão, L, entre apoios. Os cp's, de nos 1, 2 e 3, provêm de uma barra sem fios, com manta de vidro-E/epóxi com comprimento de $1200 \mathrm{~mm}$, que foi cortada em três partes com comprimentos: $115 \mathrm{~mm}, 300 \mathrm{~mm}$ e $500 \mathrm{~mm}$. Para as vigas com e sem fios de aço, realizou-se ensaios de flexão com vãos (L) entre apoios: (I) $114 \mathrm{~mm}$, (II) $150 \mathrm{~mm}$, (III) $280 \mathrm{~mm}$ e (iv) $467 \mathrm{~mm}$. Para as vigas SMAHC, ensaiaram-se nos vãos já mencionados, exceto para o de 467 $\mathrm{mm}$.

\section{ANÁLISE E DISCUSSÃO DOS RESULTADOS}

Foram ensaiados 16 cp's, com razões, L/h, no intervalo de 8 a 80. Para um viga em flexão de 3 pontos, com uma carga $\mathrm{P}$ constante no meio do vão, $\mathrm{x}=\mathrm{L} / 2$, o momento fletor máximo Mmax aumenta proporcionalmente ao vão $\mathrm{L}(\mathrm{Mmax}=\mathrm{P} . \mathrm{L} / 4)$, enquanto que o esforço cortante, $\mathrm{V}=\mathrm{P} / 2$, permanece constante. Logo, os efeitos de tensão cisalhante transversal, devido ao esforço cortante, vão se tornando desprezíveis à medida que a razão $\mathrm{L} / \mathrm{h}$ atinge valor igual ou superior a 16, de acordo com a norma ASTM D-790-08.

Nos cp's de nos 1, 2 e 3, as diferenças percentuais entre os módulos analíticos e experimentais diminuem à medida que o quociente $(\mathrm{L} / \mathrm{h})$ aumenta: de $13,61 \%$ para $11,80 \%$; decrescendo ainda mais para $1,96 \%$; até 0,34\%. Nestes casos, a razão $\mathrm{L} / \mathrm{h}=19,32$, no $\mathrm{CP} 1(\mathrm{~L}=114 \mathrm{~mm})$, aumentou no CP2 (ensaiado com os vãos: $\mathrm{L}=150 \mathrm{~mm}$; e $\mathrm{L}=280 \mathrm{~mm}$ ), e prosseguiu aumentando até $\mathrm{L} / \mathrm{h}=79,15$, no CP3 (L=467 mm), respectivamente. Portanto, a correlação teoria/experimentos melhora progressivamente com aumentos em $\mathrm{L} / \mathrm{h}$, demonstrando que o cisalhamento transversal influencia os resultados experimentais. Isto faz o Eexp aumentar quando o vão $\mathrm{L}$ aumenta e $\mathrm{h}$ permanece constante. Já os módulos experimentais a $25^{\circ} \mathrm{C}$ de vigas híbridas com fios de aço e de $\mathrm{Ni}$-Ti, respectivamente, tiveram diferenças percentuais de até $2,39 \%$ e 3,31\% inferiores aos dos analíticos, para $\mathrm{L} / \mathrm{h} \geq 16(\mathrm{~L}=280 \mathrm{~mm})$. Isto indica que as vigas híbridas fletidas a $25^{\circ} \mathrm{C}$ sofreram pouca influência do cisalhamento transversal (vide Tabela 3). Porém, para vãos menores ( $\mathrm{L}=114 \mathrm{~mm} \mathrm{e} \mathrm{L}=150 \mathrm{~mm}$ ), as diferenças percentuais aumentam consideravelmente, indicando que é importante utilizar-se $\mathrm{L} / \mathrm{h} \geq 16$ nos ensaios de flexão.

Os CP7 e CP8 foram fabricados com fios de aço galvanizado, apenas para verificar antecipadamente e aperfeiçoar os procedimentos de fabricação de vigas com fios, e notou-se nestes casos que a correlação entre resultados teóricos e experimentais, também melhora quando a razão L/h aumenta. Os cp's com fios de aço (CP8) e de Ni-Ti (CP11 e CP12, na martensita), com processos idênticos de fabricação, tiveram diferenças nos módulos analíticos na faixa de 2,50\% (CP8) a 16,84\% (CP11), ainda inferiores ao módulo do CP5 de vidro-E/epóxi. O CP5 tem o menor módulo entre os cp's de nos 1 ao 5, fabricados sem vácuo, onde os módulos variam de 11,46 a 13,97 GPa. É interessante ressaltar que o CP9 (com 4 fios) possuiu o módulo analítico na martensita ainda 6,56 \% inferior ao módulo do CP6, de apenas vidro-E/epóxi, fabricado com processos idênticos. A inclusão de camada de fios/epóxi tornou a viga como um todo menos rígida do que se tivesse no mesmo lugar uma camada de vidro-E/epóxi.

Quando foram acrescidas duas tiras de roving sobrepostas entre os fios na viga SMAHC (CP13), o módulo experimental a $25^{\circ} \mathrm{C}$ foi superior, no valor de $14,02 \%$ em relação ao módulo do CP5 para o vão de $280 \mathrm{~mm}$. As fibras de vidro-E apresentam módulo elástico de $70 \mathrm{GPa}$, enquanto que os módulos dos fios de Ni-Ti variaram de 22,6 GPa (martensita) a 48,4 GPa (austenita). Então, à medida que se aumenta a fração volumétrica de fibras de vidro-E, pode-se mascarar o efeito do aumento do módulo das vigas compósitas, com até $2 \%$ de fração volumétrica de SMA, principalmente se ainda acrescentar-se reforço de roving na camada de fios. Todas estas comparações ressaltam o fato que os materiais SMAHC devem ser utilizados como materiais funcionais em vez de estruturais [2].

Os módulos efetivos de vigas compósitas dependem tanto das frações dos constituintes, bem como de fatores geométricos não lineares, zi3/h3, onde i representa a sequência crescente do núcleo (i=1), camada de fios $(\mathrm{i}=2)$ até a camada externa (i=3) [23] . Para a seção transversal mostrada na Fig. 4, o módulo efetivo depende fortemente do fator geométrico, (z23-z13)/h3, multiplicado pelo módulo de elasticidade da camada de fios com e sem roving (vide Eq. 3). Em relação às vigas SMAHC, constatou-se o aumento de rigidez da fase martensita para a austenita de fios de Ni-Ti, para os cp's de nos 9, 13, 15 e 16, para o vão de $150 \mathrm{~mm}$, de nos 11 e 12, para o vão de $280 \mathrm{~mm}$; e também de nos 11 e 14, para o vão de $114 \mathrm{~mm}$. O que os resultados indicam é que a causa deste aumento perceptível de rigidez da viga, com fração volumétrica de até $2 \%$ de SMA, deve-se à disposição simétrica mais afastada possível das duas camadas de fios de Ni-Ti (i.e. com a coordenada z da Fig. 4 maior possível), em relação ao plano médio. Isto ocorre desta forma em função do módulo de elasticidade de cada camada, de acordo com a Eq. 3, ser multiplicado por [(z23-z13)/h3]. Deve-se 
destacar que para o CP11, com o vão de $280 \mathrm{~mm}$, o módulo de elasticidade experimental aumentou em $6,53 \%$, através da transformação de martensita para austenita dos fios de Ni-Ti, ao elevar-se a temperatura de $25^{\circ} \mathrm{C}$ para $69^{\circ} \mathrm{C}$. Foi o maior ganho de rigidez, devido ao aumento de temperatura, verificado dentre as vigas SMAHC investigadas. Nesta viga (CP11), que a frio apresentou a menor rigidez possível, não se adicionou roving na camada de fios de $\mathrm{Ni}-\mathrm{Ti}$, e a cura foi a frio e sem bolsa de vácuo.

As 16 vigas não apresentam o fenômeno da viscoelasticidade à temperatura de $25^{\circ} \mathrm{C}$ (i.e. a frio). Neste e em trabalhos anteriores na UnB, utilizando-se a mesma máquina MTS-810 [24], os cp's apresentaram comportamento linear elástico. Já em ensaios a quente, os módulos experimentais na austenita de cp's de SMAHC reduziram de $2,12 \%$ (CP9) a 17,63\% (CP16), quando a temperatura do epóxi envolvendo os fios aumentou de $25^{\circ} \mathrm{C}$ a $55^{\circ} \mathrm{C}$, para o vão de $280 \mathrm{~mm}$. Nos ensaios de flexão, tomou-se o cuidado de iniciar a aplicação da força na MTS-810 quando os fios atingiram a temperatura de $60^{\circ} \mathrm{C}$, onde a temperatura de monitoramento do epóxi dos fios marcava $45^{\circ} \mathrm{C}$. Notou-se que nos fios a $69^{\circ} \mathrm{C}$, as correlações com os experimentos pioraram para os $\mathrm{CP}^{\prime} \mathrm{s} 9,10,12,14,15$ e 16 , em relação às correlações obtidas a $25^{\circ} \mathrm{C}$, qualquer que seja o vão, principalmente para o vão $280 \mathrm{~mm}$, quando o epóxi atingiu a temperatura máxima de $55^{\circ} \mathrm{C}$. Para $\mathrm{CP} 11$, a correlação a $69^{\circ} \mathrm{C}$ piora com vão de $114 \mathrm{~mm}$ (menor) e de $280 \mathrm{~mm}$ melhora; e para o CP13, com vão de $150 \mathrm{~mm}$ (menor) piora e de $280 \mathrm{~mm}$ (maior) melhora. Isto indica que, além da influência do cisalhamento transversal nos vão menores (i.e. $\mathrm{L} / \mathrm{h}<280 \mathrm{~mm}$ ), estudos sobre a influência da temperatura nas matrizes epóxi, e na interface fio/epóxi, devem ser realizados, em trabalhos futuros.

\section{CONCLUSÕES}

Foram confeccionados cp's de compósitos híbridos com fios metálicos para a avaliação do método de fabricação proposto. Os cp's de SMAHC com diferentes processos de fabricação apresentaram forma prismática regular nos comprimentos e larguras, sempre com diferença percentuais inferiores a $1 \%$. O controle da espessura é mais difícil, porque os processos de cura, aplicando-se um peso sobre o molde macho e bolsa de vácuo, durante a fabricação, provocam alterações no volume de epóxi que é extraído da viga.

O comportamento elástico foi observado para os ensaios de flexão, com força aplicada mediante incremento monotônico, até o limite de $1 \%$ de deflexão do vão entre apoios no centro da viga. Verificou-se que as diferenças percentuais entre os módulos analíticos e experimentais foram de $0,10 \%$ a 3,31\%, em todas as vigas a $25^{\circ} \mathrm{C}$ para $\mathrm{L} / \mathrm{h} \geq 16(\mathrm{~L} \geq 280 \mathrm{~mm})$. Isto indica que quanto maior o quociente, $\mathrm{L} / \mathrm{h}$, nos ensaios de flexão a frio, menor é a influência do cisalhamento transversal. No que concerne às vigas ensaiadas a $69^{\circ} \mathrm{C}, \mathrm{o}$ melhor resultado no aumento do módulo experimental, através da transformação martensítica para a austenítica dos fios de Ni-Ti, foi de $6,53 \%$ no CP11, com o vão livre de $280 \mathrm{~mm}$. Esta viga, a $25^{\circ} \mathrm{C}$, apresenta a menor rigidez. Ela tem, em volume, menos de $2 \%$ de fios de $\mathrm{Ni}$-Ti, não possui roving e foi curada sem vácuo. Isto sugere que, mesmo com fração volumétrica de SMA inferior a $2 \%$, é possível fabricar-se uma viga SMAHC adaptativa, cuja rigidez à flexão aumente aquecendo-se os fios, sem adicionar-se roving na camada de fios e curando-a sem bolsa de vácuo. Nesta metodologia de fabricação de vigas SMAHC, os resultados indicam que os fatores que podem interferir no efeito do aumento da rigidez à flexão, na transformação dos fios de Ni-Ti, de martensita para a austenita, são: (I) as frações volumétricas de fios e de fibras de vidro-E; (II) o acréscimo ou não de roving na camada de fios; (III) o tipo de epóxi de cura a frio ou quente; (IV) a rigidez do epóxi com temperatura de ensaio muito próxima da sua temperatura de transição vítrea; e (V) o uso de bolsa com vácuo superior ou igual a 0,064 MPa.

\section{AGRADECIMENTOS}

Agradecemos à Eletronorte pela parceria com a UnB/FT/ENM na realização do projeto "Aplicação de ligas com memória de forma para controle de vibração em máquinas e estruturas" e ao CNPq.

\section{BIBLIOGRAFIA}

[1] GANDHI, M.V., THOMPSON, B.S., Smart materials and structures, London, Chapman and Hall, 1992.

[2] JANOCHA, H., Adaptronics and smart structures - basics, materials, design and applications, New York, Springer - Verlag, 1999.

[3] SRINIVASAN, A.V., MCFARLAND, M.D., Smart structures analysis and design, Cambridge, University Press, 2001.

[4] BUEHLER, W.J., GILFRICH, J.W., WILEY, R.C., "Effect of low temperature phase changes on the mechanical properties of alloys near composition TiNi”, Journal of Applied Physics, v. 34, n. 5,pp. 1475$1477,1963$. 
[5] WAYMAN, C.M., DUERIG, T.W., Engineering aspects of shape memory alloys., Oxford, ButterworthHeinemann, 1990.

[6] OTSUKA, K., WAYMAN, C.M., Shape memory materials, Cambridge, University Press, 1998.

[7] ROGERS, C.A., ROBERTSHAW, H.H., "Shape memory reinforced composites", Engineering Science Preprints 25, ESP25.88027, Society of Engineering Sciences, 1988.

[8] BRISON, L.C., HUANG, M.S., BOLLER, C., et al., "Analysis of controlled beam deflections using SMA wires”, Journal of Intelligent Material Systems and Structures, v. 8, n. 2, pp. 12-25, Jan. 1997.

[9] BRINSON, L.C., "Simplifications and comparisons of shape memory alloy constitutive models", Journal of Intelligent Material Systems and Structures, v. 7, n. 1, pp.108 - 114, Jan. 1996.

[10] TURNER, T.L., "Thermomechanical response of shape memory alloy hybrid composites", NASA/TM2001-210656, Langley Research Center, Hampton, Virginia, 2001.

[11] SUN, G., SUN, C.T., "One-dimensional constitutive relation for shape-memory alloy-reinforced composite lamina”, Journal of Materials Science, v. 28, n. 23, pp. 6323-6328, 1993.

[12] PARTHENIOS, G.C., PSARRAS, C.G., "Adaptative incorporating shape memory alloy wires. Part 2: Development of internal recovery stresses as a function of activation temperature", Composites: Part A applied science and manufacturing, v. 32, n.12, pp. 1735-1747, Jan. 2001.

[13] ZAK, A.J., CARTMELL, M.P., OSTACHOWICZ, W.M., "Dynamics and control of a rotor using an integrated SMA/composite active bearing actuator”, Key Engineering Materials, v. 245-246, pp. 233-240, Jul. 2003.

[14] TZOI, K.A., SCHROOTEN, J., ZHENG, Y., et al., "Part II. Thermomechanical characteristics of shape memory alloy composites”, Materials Science and Engineering: A, v. 368, n.1-2, pp. 299-310, 2004.

[15] XU, J., OTSUKA, N., TOYAMA, N., et al., "A novel technique for fabricating SMA/CFRP adaptative composites using ultrathin TiNi wires”, Smart Materials and Structures, v. 13, n.1, pp. 196-202, 2004.

[16] YUSE, K., KIKUSHIMA, Y., "Development and experimental consideration of SMA/CFRP actuator for vibration control”, Sensors and Actuators A: phisycal, v. 122, n.1, pp.99-107, March 2005.

[17] BAZ, A., IMAN, K., MCCOY, J., “Active vibration control of flexible beams using shape memory actuators", Journal of Sound and Vibration, v. 140, n.3, pp. 457-456, 1990.

[18] SHIMAMOTO, A., OHKAWARA, H., NOGATA, F., "Enhancement of mechanical strength by shape memory effect in TiNi fiber-reinforced composites", Engineering Fracture Mechanics, v. 71, n.4-6, pp. 737746, Nov. 2002.

[19] AOKI, T., SHIMAMOTO, A., “Active vibration control using cantilever beam of smart matrix composite with embedded shape memory alloy”, Key Engineering Materials, v. 270-273, pp. 2187-2192, 2004.

[20] FALUHELYI, P., Fabricação e comportamento termomecânico de compósitos estruturais adaptativos com filamentos de Ni-Ti, Tese de D.Sc., Departamento de Engenharia Mecânica/UnB, Distrito Federal, Brasília, 2010.

[21] JIA, J., ROGERS, C.A., "Formulation of a mechanical model for composites with embedded SMA actuators", In: Proceedings of the Eighth Biennial Conference: Failure Prevention and Reliability, Montreal, Canada, pp.203-210, 1989

[22] CERÓN, D.M.S., Desenvolvimento de uma metodologia para fabricação de compósitos híbridos com memória de forma, Dissertação de M.Sc em Ciências Mecânicas, Departamento de Engenharia Mecânica, Universidade de Brasília, Brasília, DF, 2010.

[23] LEVY-NETO, F., PARDINI, L.C., Compósitos Estruturais, Ciência e Tecnologia, 1 ed., São Paulo, Edgard Blücher, 2006.

[24] FALUHELYI, P., LEVY-NETO, F., SILVA, E.P. da, "Mechanical behavior of SMAHC bars and beams tested at two different temperatures", In: Brazilian Congress of Mechanical Engineering (COBEM), 21st , Brazil, 2011.

[25] MENDONÇA, P.T.R., Materiais Compostos e Estruturas-Sanduíche, 1 ed, cap.7, Santa Catarina, Editora Manole, 2005. 